\title{
Author Correction: GWAS for male-pattern baldness identifies 71 susceptibility loci explaining $38 \%$ of the risk
}

\author{
Nicola Pirastu (10 1, Peter K. Joshi (10 1, Paul S. de Vries ${ }^{2}$, Marilyn C. Cornelis ${ }^{3}$, Paul M. McKeigue ${ }^{4}$, NaNa Keum ${ }^{5,6}$, \\ Nora Franceschini ${ }^{7}$, Marco Colombo ${ }^{4}$, Edward L. Giovannucci ${ }^{6,8,9}$, Athina Spiliopoulou ${ }^{4,10}$, Lude Franke (1D ${ }^{11}$, \\ Kari E. North7 ${ }^{7}$ Peter Kraft ${ }^{12}$, Alanna C. Morrison², Tõnu Esko (iD ${ }^{13,14}$ \& James F. Wilson (iD ${ }^{1,15}$
}

Correction to: Nature Communications https://doi.org/10.1038/s41467-017-01490-8, published online 17 November 2017.

We have been alerted ${ }^{1}$ that in our recent Article $^{2}$ the calculations used to transform the heritability from the observed scale to the liability scale did not take into account the individuals in category 2 of the baldness scale, who were removed in our original analysis. This led to an overestimation of the heritability on the liability scale, which should have been 0.62 instead of 0.94 . Moreover, in the Title and in the Abstract, we report that we can explain $38 \%$ of the risk, while in fact that is the proportion of heritability explained by the loci we discovered. These errors do not substantially change the paper or its conclusions apart from the statement MBP is therefore probably one of the most heritable complex traits. Genome-wide significant associations and pathway analyses are not affected in any way and male-pattern baldness remains less genetically complex than other complex traits. We wish to thank Yap et al. for bringing this to our attention.

Published online: 29 June 2018

\section{References}

1. Yap C. X. et al. Correspondence: mis-estimation of heritability and prediction accuracy of male-pattern baldness. Nat Commun. 2018; https://doi.org/10.1038/s41467018-04807-3

2. Pirastu, N., Joshi, P. K. \& de Vries, P. S. et al. GWAS for male-pattern baldness identifies 71 susceptibility loci explaining $38 \%$ of the risk. Nat. Commun. 8, 1584 (2017).

(i) Open Access This article is licensed under a Creative Commons Attribution 4.0 International License, which permits use, sharing, adaptation, distribution and reproduction in any medium or format, as long as you give appropriate credit to the original author(s) and the source, provide a link to the Creative Commons license, and indicate if changes were made. The images or other third party material in this article are included in the article's Creative Commons license, unless indicated otherwise in a credit line to the material. If material is not included in the article's Creative Commons license and your intended use is not permitted by statutory regulation or exceeds the permitted use, you will need to obtain permission directly from the copyright holder. To view a copy of this license, visit http://creativecommons.org/licenses/by/4.0/.

\footnotetext{
${ }^{1}$ Centre for Global Health Research, Usher Institute of Population Health Sciences and Informatics, University of Edinburgh, Teviot Place, Edinburgh EH8 9 AG, Scotland. ${ }^{2}$ Human Genetics Center, Department of Epidemiology, Human Genetics and Environmental Sciences, School of Public Health, The University of Texas Health Science Center at Houston, Houston, TX 77030, USA. ${ }^{3}$ Department of Preventive Medicine, Northwestern University Feinberg School of Medicine, Chicago, IL 60611, USA. ${ }^{4}$ Centre for Population Health Sciences, Usher Institute of Population Health Sciences and Informatics, University of Edinburgh, Teviot Place, Edinburgh EH8 9AG, Scotland. ${ }^{5}$ Department of Food Science and Biotechnology, Dongguk University, Goyang, South Korea. ${ }^{6}$ Department of Nutrition, Harvard T. H. Chan School of Public Health, Boston, MA 02115, USA. ${ }^{7}$ Department of Epidemiology and Carolina Center for Genome Sciences, University of North Carolina, Chapel Hill, NC 27599, USA. ${ }^{8}$ Department of Epidemiology, Harvard T. H. Chan School of Public Health, Boston, MA 0211, USA. ${ }^{9}$ Channing Division of Network Medicine, Department of Medicine, Brigham and Women's Hospital and Harvard Medical School, Boston, MA 02115, USA. ${ }^{10}$ Pharmatics Ltd, Edinburgh EH16 4UX, Scotland. ${ }^{11}$ Department of Genetics, University Medical Center, 9713 GZ Gröningen, The Netherlands. ${ }^{12}$ Program in Genetic Epidemiology and Statistical Genetics, Harvard T. H. Chan School of Public Health, Boston, MA 02115, USA. ${ }^{13}$ Estonian Genome Center, University of Tartu, Tartu 51010, Estonia. ${ }^{14}$ Broad Institute of Harvard and MIT, Cambridge, MA 02142, USA. ${ }^{15}$ MRC Human Genetics Unit, Institute of Genetics and Molecular Medicine, University of Edinburgh, Western General Hospital, Crewe Road, Edinburgh EH4 2XU, Scotland.

Correspondence and requests for materials should be addressed to N.P. (email: nicola.pirastu@ed.ac.uk)
} 